\title{
Carotid-carotid bypass for a carotid artery aneurysm
}

Yoichi Morofuji, MD, PhD, Yuki Matsunaga, MD, Tsuyoshi Izumo, MD, PhD

Departments of Neurosurgery, Nagasaki University Graduate School of Biomedical

Sciences, 1-7-1 Sakamoto, Nagasaki 852-8501 Japan

\section{Address correspondence and reprint requests to:}

Yoichi Morofuji, MD, PhD, Department of Neurosurgery, Nagasaki University School of

Medicine, 1-7-1 Sakamoto, Nagasaki 852-8501, Japan.

Tel: 81-095-819-7375, Fax: 81-095-819-7378

E-mail: yoichi51@hotmail.com

Key words: bypass, extracranial carotid artery aneurysm, saphenous vein graft

Short title: carotid-carotid bypass

Declarations of interest: none 


\author{
Abbreviations list \\ Computed tomography: CT \\ common carotid artery: CCA \\ saphenous vein: SV
}

\begin{abstract}
A 55-year-old man presented with progressive right-sided hemiparesis and dysarthria.

Computed tomography angiography revealed the cause of his repeated cerebral

infarction was due to the distal emboli from an intra-aneurysmal thrombus of a giant

thrombosed aneurysm at the origin of left common carotid artery. Right common carotid artery-saphenous vein graft-left common carotid artery bypass followed by left common carotid artery ligation was successfully performed. Carotid-carotid bypass followed by common carotid artery ligation is an optional procedure for symptomatic proximal common carotid artery aneurysm.
\end{abstract}

\title{
Clinical Images
}

A 55-year-old man presented with progressive right-sided hemiparesis and dysarthria.

Serial Magnetic resonance imaging showed increasing multiple small infarction in his 
left hemisphere. Computed tomography (CT) angiography revealed a giant thrombosed aneurysm at the origin of left common carotid artery (CCA). The cause of his repeated cerebral infarction was due to the distal emboli from an intra-aneurysmal thrombus. Parent artery occlusion by surgical ligation or endovascular approach without bypass might have a risk of ischemic complications and endovascular treatment including covered stent or overlapping stent placement was not suitable because proper placement of guiding catheter was difficult as the aneurysm was located at the proximal portion of CCA. Right CCA-saphenous vein (SV) graft-left CCA bypass followed by left CCA ligation was performed. A subcutaneous tunnel was made by blunt dissection across neck in a plane anterior to the trachea. The saphenous vein graft was tunneled in a gentle curve, ensuring placement without kinks. The patency of the bypass under temporary clipping of left CCA was confirmed by intra-operative digital subtraction angiography. The postoperative was uneventful, patient's symptoms gradually recovered. One week after the surgery, the patency of the bypass and normal perfusion were observed on CT angiography.

Surgical treatment for symptomatic extracranial carotid artery aneurysms should be encouraged because of their high recurrence rates. ${ }^{1,2}$ Parent artery occlusion is technically easy and suitable for the patients with ischemic tolerance, however, balloon 
occlusion test itself has a risk of complications, especially during acute stroke stage. ${ }^{3}$

Furthermore, high morbidity rate with transthoracic surgery for lesions in the common

carotid arteries was reported. ${ }^{4}$

In conclusions, carotid-carotid bypass followed by CCA ligation is an optional

procedure for symptomatic proximal CCA aneurysm.

Competing interests: None declared.

Authors' contributions: Conception and Design: Morofuji, Izumo. Acquisition of data:

Matsunaga. Drafting the article: Morofuji. Critically revising the article: all authors.

\section{Acknowledgements}

We wish to thank Takeo Naito, Nobutaka Horie, Takayuki Matsuo for providing insight and expertise that assisted this paper.

\section{Funding:}

This work was supported by a Grant-in-Aid for Scientific Research (grant number 17K10840).

\section{References}

1. Zwolak RM, Whitehouse WM, Jr., Knake JE, et al: Atherosclerotic extracranial 
carotid artery aneurysms. J Vasc Surg 1984;1(3):415-422.

2. Li Z, Chang G, Yao C, Guo L, et al. Endovascular stenting of extracranial carotid artery aneurysm: a systematic review. Eur J Vasc Endovasc Surg 2011;42(4):419-426. https://doi.org/10.1016/j.ejvs.2011.05.008

3. Mathis JM, Barr JD, Jungreis CA, et al. Temporary balloon test occlusion of the internal carotid artery: experience in 500 cases. AJNR Am J Neuroradiol 1995;16(4):749-754.

4. Berguer R, Morasch MD, Kline RA. Transthoracic repair of innominate and common carotid artery disease: immediate and long-term outcome for $\mathbf{1 0 0}$ consecutive surgical reconstructions. J Vasc Surg 1998;27(1):34-41; discussion 42.

\section{Figure legends}

Figure. (A) Computed tomography (CT) angiography shows a giant thrombosed aneurysm at the origin of left common carotid artery (arrows). (B) Intraoperative digital subtraction angiography shows a good flow between right common carotid artery (CCA) to left CCA through saphenous vein graft (arrowhead). (C) CT angiography a week after the surgery shows the patent bypass flow and disappearance of the aneurysm. 
Figure

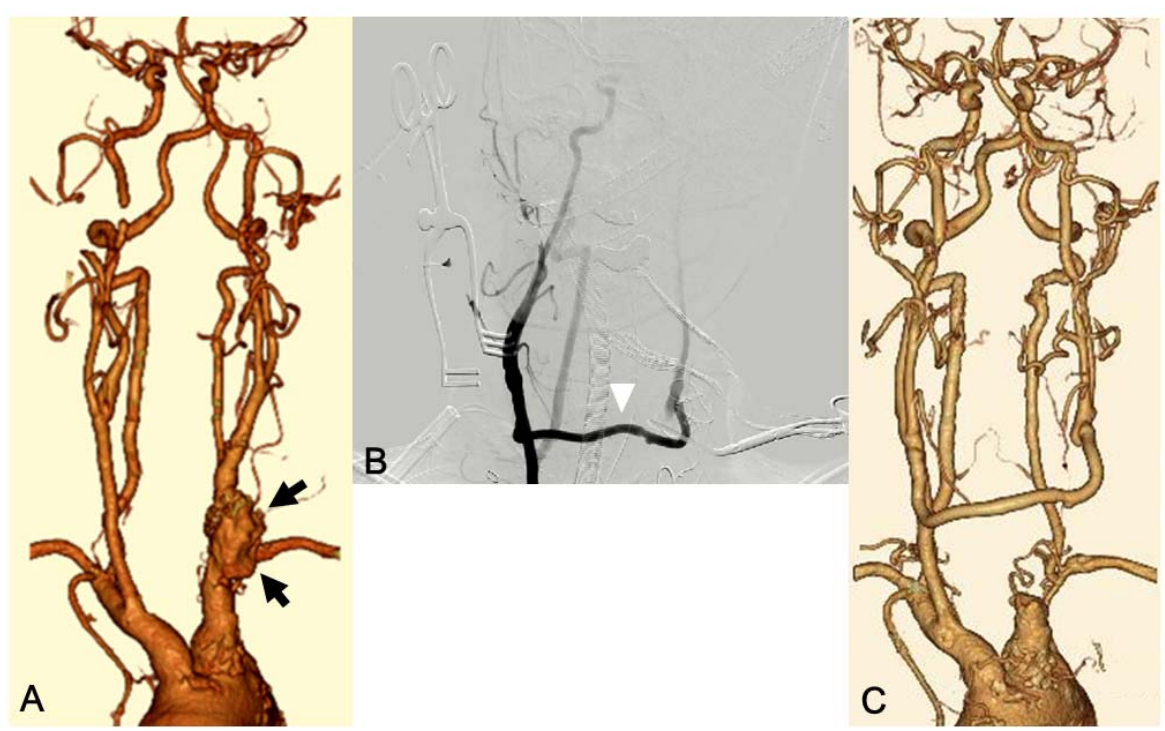

